\title{
GRAVITINO PRODUCTION BY PRIMORDIAL BLACK HOLE EVAPORATION AND CONSTRAINTS ON THE INHOMOGENEITY OF THE EARLY UNIVERSE
}

\author{
M. Yu. Khlopov \\ Physics Department, Rome University 1 "La Sapienza" \\ P. le A. Moro, 2, 00185, Rome, Italy \\ Cosmion, Keldysh Institute of Applied Mathematics \\ Miusskaya pl. 4, 125047, Moscow, Russia \\ Moscow Engineering Physics Institute \\ Kashirskoe shosse 31, 115409, Moscow, Russia \\ A. Barrau, J. Grain \\ Laboratory for Subatomic Physics and Cosmology \\ Joseph Fourier University, CNRS-IN2P3 \\ 53, avenue des Martyrs, 38026 Grenoble cedex, France
}

\begin{abstract}
In supergravity models, the evaporation of light Primordial Black Holes $(\mathrm{PBHs})$ should be a source of gravitinos. By considering this process, new stringent limits are derived on the abundance of small black holes with initial masses less than $10^{9} \mathrm{~g}$. In minimal supergravity, the subsequent decay of evaporated gravitinos into cascades of non-equilibrium particles leads to the formation of elements whose abundance is constrained by observations. In gauge mediated supersymmetry breaking models, their density is required not to overclose the Universe. As a result, cosmological models with substantial inhomogeneities on small scales are excluded.
\end{abstract}




\section{Introduction}

Primordial Black Holes (PBHs) are a very sensitive cosmological probe for physics phenomena occurring in the early Universe. They could be formed by many different mechanisms, e.g., initial density inhomogeneities [1, a softening of the equation of state [2, 3, 4, collapse of cosmic strings [5], a double inflation scenario [6], phase transitions [7], a step in the power spectrum [8], etc. (see [9] for a review). Constraining the abundance of primordial black holes can lead to invaluable information on cosmological processes, particularly as they are probably the only viable probe for the power spectrum on very small scales which remain far from the Cosmological Microwave Background (CMB) and Large Scale Structures (LSS) sensitivity ranges. To date, only PBHs with initial masses between $\sim 10^{9} \mathrm{~g}$ and $\sim 10^{16}$ g have led to stringent limits (see e.g. [10, 11]) from consideration of the entropy per baryon, the deuterium destruction, the ${ }^{4} \mathrm{He}$ destruction and the cosmic-rays currently emitted by the Hawking process [12]. The existence of light PBHs should lead to important observable constraints, either through the direct effects of the evaporated particles (for initial masses between $10^{14} \mathrm{~g}$ and $10^{16} \mathrm{~g}$ ) or through the indirect effects of their interaction with matter and radiation in the early Universe (for $\mathrm{PBH}$ masses between $10^{9} \mathrm{~g}$ and $10^{14} \mathrm{~g}$ ). In these constraints, the effects taken into account are those related with known particles. However, since the evaporation products are created by the gravitational field, any quantum with a mass lower than the black hole temperature should be emitted, independently of the strength of its interaction. This could provide a copious production of superweakly interacting particles that cannot not be in equilibrium with the hot plasma of the very early Universe.

Following [9, 13, 14] and [15, 16] (but in a different framework and using more stringent constraints), this article derives new limits on the mass fraction of black holes at the time of their formation $\left(\beta \equiv \rho_{P B H} / \rho_{t o t}\right)$ using the production of gravitinos during the evaporation process. Depending on whether gravitinos are expected to be stable or metastable, the limits are obtained using the requirement that they do not overclose the Universe and that the formation of light nuclei by the interactions of ${ }^{4} \mathrm{He}$ nuclei with nonequilibrium flux of $\mathrm{D}, \mathrm{T},{ }^{3} \mathrm{He}$ and ${ }^{4} \mathrm{He}$ does not contradict the observations. This approach is more constraining than the usual study of photo-dissociation induced by photons-photinos pairs emitted by decaying gravitinos. It opens a new window for the upper limits on $\beta$ below $10^{9} \mathrm{~g}$. The cosmological consequences of our new limits are briefly reviewed in the framework of 3 different scenarios : a blue power spectrum, a step in the power spectrum and first order phase transitions.

\section{New limits on the PBH density}

Several constraints on the density of PBHs have been derived in different mass ranges assuming the evaporation of only standard model particles : for $10^{9} \mathrm{~g}<$ 
$M<10^{13} \mathrm{~g}$ the entropy per baryon at nucleosynthesis was used [17 to obtain $\beta<\left(10^{9} \mathrm{~g} / M\right)$, for $10^{9} \mathrm{~g}<M<10^{11} \mathrm{~g}$ the production of $n \bar{n}$ pairs at nucleosynthesis was used [18] to obtain $\beta<3 \times 10^{-17}\left(10^{9} \mathrm{~g} / M\right)^{1 / 2}$, for $10^{10} \mathrm{~g}<M<10^{11} \mathrm{~g}$ deuterium destruction was used [19] to obtain $\beta<3 \times 10^{-22}\left(M / 10^{10} \mathrm{~g}\right)^{1 / 2}$, for $10^{11} \mathrm{~g}<M<10^{13} \mathrm{~g}$ spallation of ${ }^{4} \mathrm{He}$ was used [20, 13] to obtain $\beta<$ $3 \times 10^{-21}\left(M / 10^{9} \mathrm{~g}\right)^{5 / 2}$, for $M \approx 5 \times 10^{14} \mathrm{~g}$ the gamma-rays and cosmic-rays were used 21, 22 to obtain $\beta<10^{-28}$. Slightly more stringent limits where obtained in [23], leading to $\beta<10^{-20}$ for masses between $10^{9} \mathrm{~g}$ and $10^{10} \mathrm{~g}$ and in 24, leading to $\beta<10^{-28}$ for $M=5 \times 10^{11} \mathrm{~g}$. Gamma-rays and antiprotons where also recently re-analyzed in [25] and [26], improving a little the previous estimates. Such constraints, related to phenomena occurring after the nucleosynthesis, apply only for black holes with initial masses above $\sim 10^{9} \mathrm{~g}$. Below this value, the only limits are the very weak entropy constraint (related with the photonto-baryon ratio) and the quite doubtful relics constraint (assuming stable black holes forming at the end of the evaporation mechanism as described, e.g., in [27]).

To derive a new limit in the initial mass range $M_{P l}<M<10^{11} \mathrm{~g}$, gravitinos emitted by black holes are considered in this work. Gravitinos are expected to be present in all local supersymmetric models, which are regarded as the more natural extensions of the standard model of high energy physics (see, e.g., [28] for an introductory review). In the framework of minimal Supergravity (mSUGRA), the gravitino mass is, by construction, expected to lie around the electroweak scale, i.e. in the $100 \mathrm{GeV}$ range. In this case, the gravitino is metastable and decays after nucleosynthesis, leading to important modifications of the nucleosynthesis paradigm. Instead of using the usual photon-photino decay channel, this study relies on the more sensitive gluon-gluino channel. Based on [29], the antiprotons produced by the fragmentation of gluons emitted by decaying gravitinos are considered as a source of nonequilibrium light nuclei resulting from collisions of those antiprotons on equilibrium nuclei. Then, ${ }^{6} \mathrm{Li},{ }^{7} \mathrm{Li}$ and ${ }^{7} \mathrm{Be} \mathrm{nu}-$ clei production by the interactions of the nonequilibrium nuclear flux with ${ }^{4} \mathrm{He}$ equilibrium nuclei is taken into account and compared with data (this approach is supported by several recent analysis [30] which lead to similar results). The resulting Monte-Carlo estimates [29] lead to the following constraint on the concentration of gravitinos: $n_{3 / 2}<1.1 \times 10^{-13} m_{3 / 2}^{-1 / 4}$ where $m_{3 / 2}$ is the gravitino mass in $\mathrm{GeV}$. This constraint has been successfully used to derive an upper limit on the reheating temperature of the order [29]: $T_{R}<3.8 \times 10^{6} \mathrm{GeV}$. The consequences of this limit on cosmic-rays emitted by PBHs was considered, e.g., in [31. In the present approach, we relate this stringent constraint on the gravitino abundance to the density of PBHs through the direct gravitino emission. The usual Hawking formula [12] is used for the number of particles of type $i$ emitted per unit of time $t$ and per unit of energy $Q$. Introducing the temperature defined by $T=h c^{3} /(16 \pi k G M) \approx\left(10^{13} \mathrm{~g}\right) / M \mathrm{GeV}$, taking the relativistic approximation for $\Gamma_{s}$, and integrating over time and energy, the total number of quanta of type 
$i$ can be estimated as:

$$
N_{i}^{T O T}=\frac{27 \times 10^{24}}{64 \pi^{3} \alpha_{S U G R A}} \int_{T_{i}}^{T_{P l}} \frac{d T}{T^{3}} \int_{m / T}^{x} \frac{x^{2} d x}{e^{x}-(-1)^{s}}
$$

where $T$ is in $\mathrm{GeV}, M_{P l} \approx 10^{-5} \mathrm{~g}, x \equiv Q / T, m$ is the particle mass and $\alpha_{S U G R A}$ accounts for the number of degrees of freedom through $M^{2} d M=-\alpha_{S U G R A} d t$ where $M$ is the black hole mass. Once the $\mathrm{PBH}$ temperature is higher than the gravitino mass, gravitinos will be emitted with a weight related with their number of degrees of freedom. Computing the number of emitted gravitinos as a function of the $\mathrm{PBH}$ initial mass and matching it with the limit on the gravitino density imposed by nonequilibrium nucleosynthesis of light elements leads to an upper limit on the $\mathrm{PBH}$ number density. If $\mathrm{PBH}$ are formed during a radiation dominated stage, this limit can easily be converted into an upper limit on $\beta$ by evaluating the energy density of the radiation at the formation epoch. The resulting limit is shown on Fig. 1 and leads to an important improvement over previous limits, nearly independently of the gravitino mass in the interesting range. This opens a new window on the very small scales in the early Universe.

It is also possible to consider limits arising in Gauge Mediated Susy Breaking (GMSB) models 32. Those alternative scenarios, incorporating a natural suppression of the rate of flavor-changing neutral-current due to the low energy scale, predict the gravitino to be the Lightest Supersymmetric Particle (LSP). The LSP is stable if R-parity is conserved. In this case, the limit is obtained by requiring $\Omega_{3 / 2,0}<\Omega_{M, 0}$, i.e. by requiring that the current gravitino density does not exceed the matter density. It can easily be derived from the previous method, by taking into account the dilution of gravitinos in the period of $\mathrm{PBH}$ evaporation and conservation of gravitino to specific entropy ratio, that:

$$
\beta \leq \frac{\Omega_{M, 0}}{N_{3 / 2} \frac{m_{3 / 2}}{M}\left(\frac{t_{e q}}{t_{f}}\right)^{\frac{1}{2}}}
$$

where $N_{3 / 2}$ is the total number of gravitinos emitted by a $\mathrm{PBH}$ with initial mass $M, t_{e q}$ is the end of $\mathrm{RD}$ stage and $t_{f}=\max \left(t_{\text {form }}, t_{\text {end }}\right)$ when a non-trivial equation of state for the period of $\mathrm{PBH}$ formation is considered, e.g. a dust-like phase which ends at $t_{\text {end }}$ [33. The limit does not imply thermal equilibrium of relativistic plasma in the period before $\mathrm{PBH}$ evaporation and is valid even for low reheating temperatures provided that the equation of state on the preheating stage is close to relativistic. With the present matter density $\Omega_{M, 0} \approx 0.2735$ ] this leads to the limits shown in Fig. 2 for two extreme cases: $m_{3 / 2}=10^{-5} \mathrm{GeV}$ and $m_{3 / 2}=10 \mathrm{GeV}$. These results are close to the previous ones and remain very competitive in this mass range. Models of gravitino dark matter with $\Omega_{3 / 2,0}=$ $\Omega_{C D M, 0}$, corresponding to the case of equality in the above formula, were recently considered in 34. 


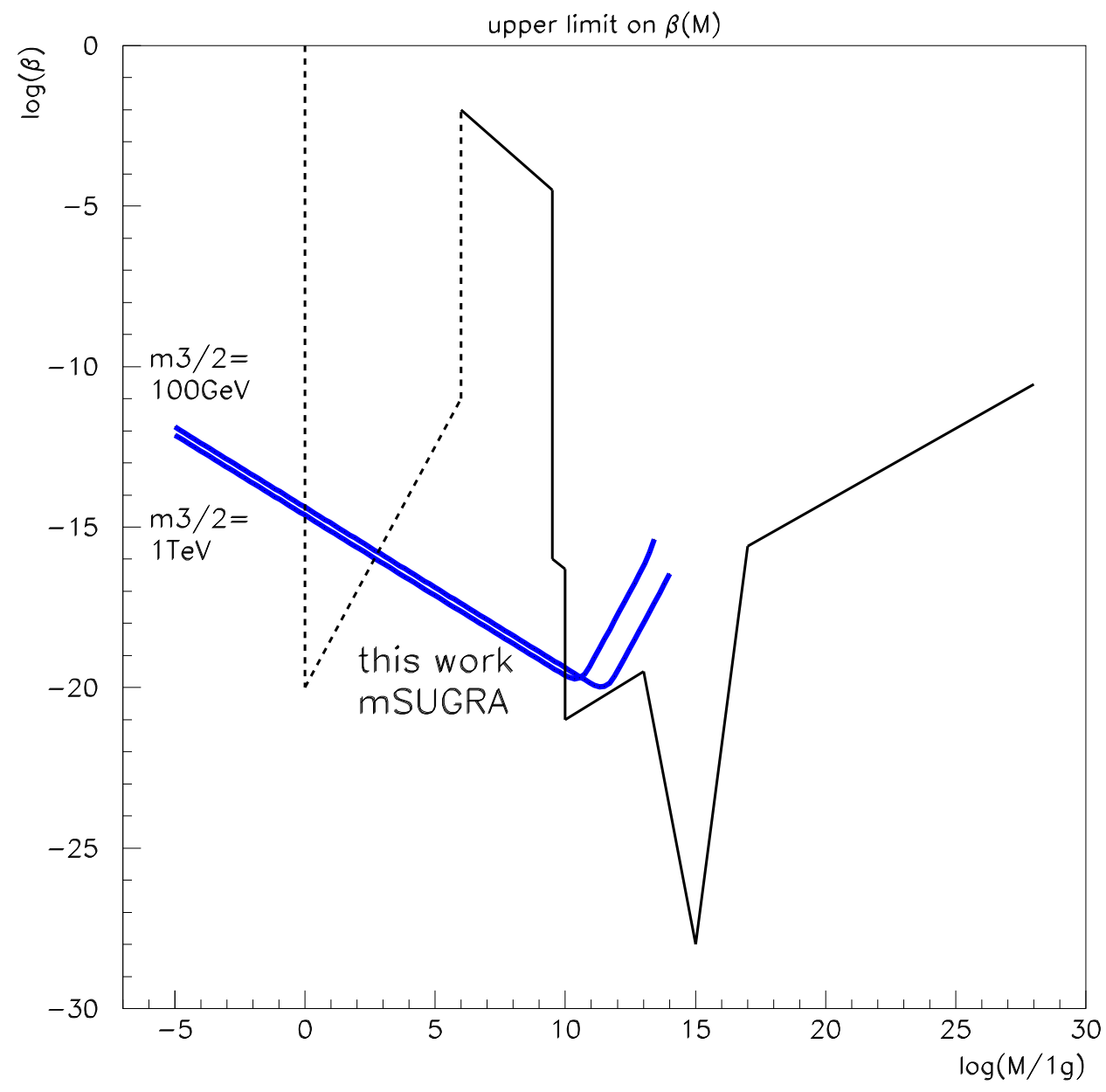

Figure 1: Constraints on the fraction of the Universe going into PBHs (adapted from [10, 11]). The two curves obtained with gravitinos emission in mSUGRA correspond to $m_{3 / 2}=100 \mathrm{GeV}$ (lower curve in the high mass range) and $m_{3 / 2}=$ $1 \mathrm{TeV}$ (upper curve in the high mass range) 


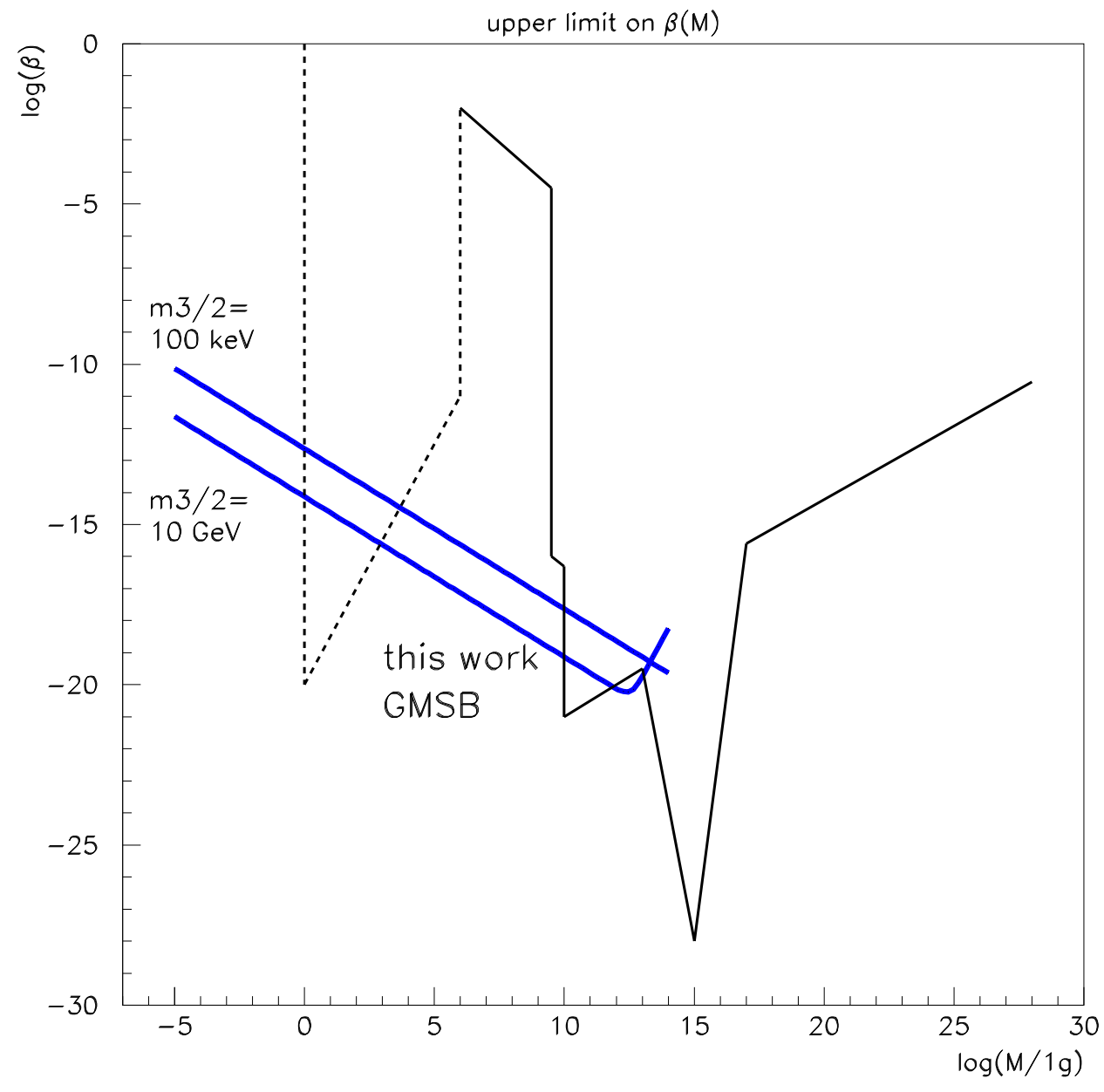

Figure 2: Constraints on the fraction of the Universe going into PBHs (adapted from [10, 11]). The two curves obtained with gravitinos emission in GMSB correspond to $m_{3 / 2}=10^{-5} \mathrm{GeV}$ (lower curve in the high mass range) and $m_{3 / 2}=10$ $\mathrm{GeV}$ (upper curve in the high mass range) 


\section{Cosmological consequences}

Our new stringent upper limits on the fraction of the Universe in primordial black holes can be converted into cosmological constraints on models with significant power on small scales.

The easiest way to illustrate the importance of such limits is to consider a blue power spectrum and to derive a related upper value on the spectral index $n$ of scalar fluctuations $\left(P(k) \propto k^{n}\right)$. It has recently been shown by WMAP [35] that the spectrum is nearly of the Harrison-Zel'dovich type, i.e. scale invariant with $n \approx 1$. However this measure was obtained for scales between $10^{45}$ and $10^{60}$ times larger that those probed by $\mathrm{PBH}$ and it remains very important to probe the power available on small scales. The limit on $n$ given in this paper must therefore be understood as a way to constrain $P(k)$ at small scales rather than a way to measure its derivative at large scales : it is complementary to CMB measurements. Using the usual relations between the mass variance at the $\mathrm{PBH}$ formation time $\sigma_{H}\left(t_{\text {form }}\right)$ and the same quantity today $\sigma_{H}\left(t_{0}\right)$ [36],

$$
\sigma_{H}\left(t_{\text {form }}\right)=\sigma_{H}\left(t_{0}\right)\left(\frac{M_{H}\left(t_{0}\right)}{M_{H}\left(t_{\text {eq }}\right)}\right)^{\frac{n-1}{6}}\left(\frac{M_{H}\left(t_{\text {eq }}\right)}{M_{H}\left(t_{\text {form }}\right)}\right)^{\frac{n-1}{4}}
$$

where $M_{H}(t)$ is the Hubble mass at time $t$ and $t_{e q}$ is the equilibrium time, it is possible to set an upper value on $\beta$ which can be expressed as

$$
\beta \approx \frac{\sigma_{H}\left(t_{\text {form }}\right)}{\sqrt{2 \pi} \delta_{\text {min }}} e^{-\frac{\delta_{\min }^{2}}{2 \sigma_{H}^{2}\left(t_{\text {form }}\right)}}
$$

where $\delta_{\text {min }} \approx 0.3$ is the minimum density contrast required to form a $\mathrm{PBH}$. The limit derived in the previous section leads to $n<1.20$ in the mSUGRA case whereas the usually derived limits range between 1.23 and 1.31 [36, 37. In the GMSB case, it remains at the same level for $m_{3 / 2} \sim 10 \mathrm{GeV}$ and is slightly relaxed to $n<1.21$ for $m_{3 / 2} \sim 100 \mathrm{keV}$. This substantial improvement is due to the much more important range of masses probed and where derived for $M \sim 1 \mathrm{~g}$.

In the standard cosmological paradigm of inflation, the primordial power spectrum is expected to be nearly -but not exactly- scale invariant [41. The sign of the running can, in principle, be either positive or negative. It has been recently shown that models with a positive running $\alpha_{s}$, defined as

$$
P(k)=P\left(k_{0}\right)\left(\frac{k}{k_{0}}\right)^{n_{s}\left(k_{0}\right)+\frac{1}{2} \alpha_{s} \ln \left(\frac{k}{k_{0}}\right)},
$$

are very promising in the framework of supergravity inflation (see, e.g., 42]). Our analysis strongly limits a positive running, setting the upper bound at a tiny value $\alpha_{s}<2 \times 10^{-3}$. This result is more stringent than the upper limit 
obtained through a combined analysis of Ly $\alpha$ forest, SDSS and WMAP data 43], $-0.013<\alpha_{s}<0.007$, as it deals with scales very far from those probed by usual cosmological observations. The order of magnitude of the running naturally expected in most models - either inflationary ones (see, e.g., 44) or alternative ones (see, e.g., 45]) - being of a few times $10^{-3}$ our upper bound should help to distinguish between different scenarios.

In the case of an early dust-like stage in the cosmological evolution [3, 11, 9], the $\mathrm{PBH}$ formation probability is increased to $\beta>\delta^{13 / 2}$ where $\delta$ is the density contrast for the considered small scales. The associated limit on $n$ is strengthened to $n<1.19$.

Following [36, it is also interesting to consider primordial density perturbation spectra with both a tilt and a step. Such a feature can arise from underlying physical processes [38] and allows investigation of a wider class of inflaton potentials. If the amplitude of the step is defined so that the power on small scales is $p^{-2}$ times higher than the power on large scales, the maximum allowed value for the spectral index can be computed as a function of $p$. Figure 3 shows those limits, which become extremely stringent when $p$ is small enough, for both the radiation-dominated and the dust-like cases. The different values of the gravitino mass considered in the first section of this paper are all included within the width of the lines.

Another important consequence of our new limits concerns PBH relics dark matter. The idea, introduced in [39], that relics possibly formed at the end of the evaporation process could account for the cold dark matter has been extensively studied. The amplitude of the power boost required on small scales has been derived, e.g., in [40] as a function of the relic mass and of the expected density. The main point was that the "step" (or whatever structure in the power spectrum) should occur at low masses to avoid the constraints available between $10^{9} \mathrm{~g}$ and $10^{15} \mathrm{~g}$. The limit on $\beta$ derived in this work closes this dark matter issue except within a small window below $10^{3} \mathrm{~g}$.

Finally, the limits also completely exclude the possibility of a copious PBH formation process in bubble wall collisions [46. This has important consequences for the related constraints on first order phase transitions in the early Universe.

\section{Conclusion and prospects}

If local supersymmetry - supergravity - is the correct extension of the standard model, the emission of gravitinos from evaporating primordial black holes leads to important constraints on their number density in the - so far nearly unexplored - low formation mass range. This allows us to exclude cosmological models with 


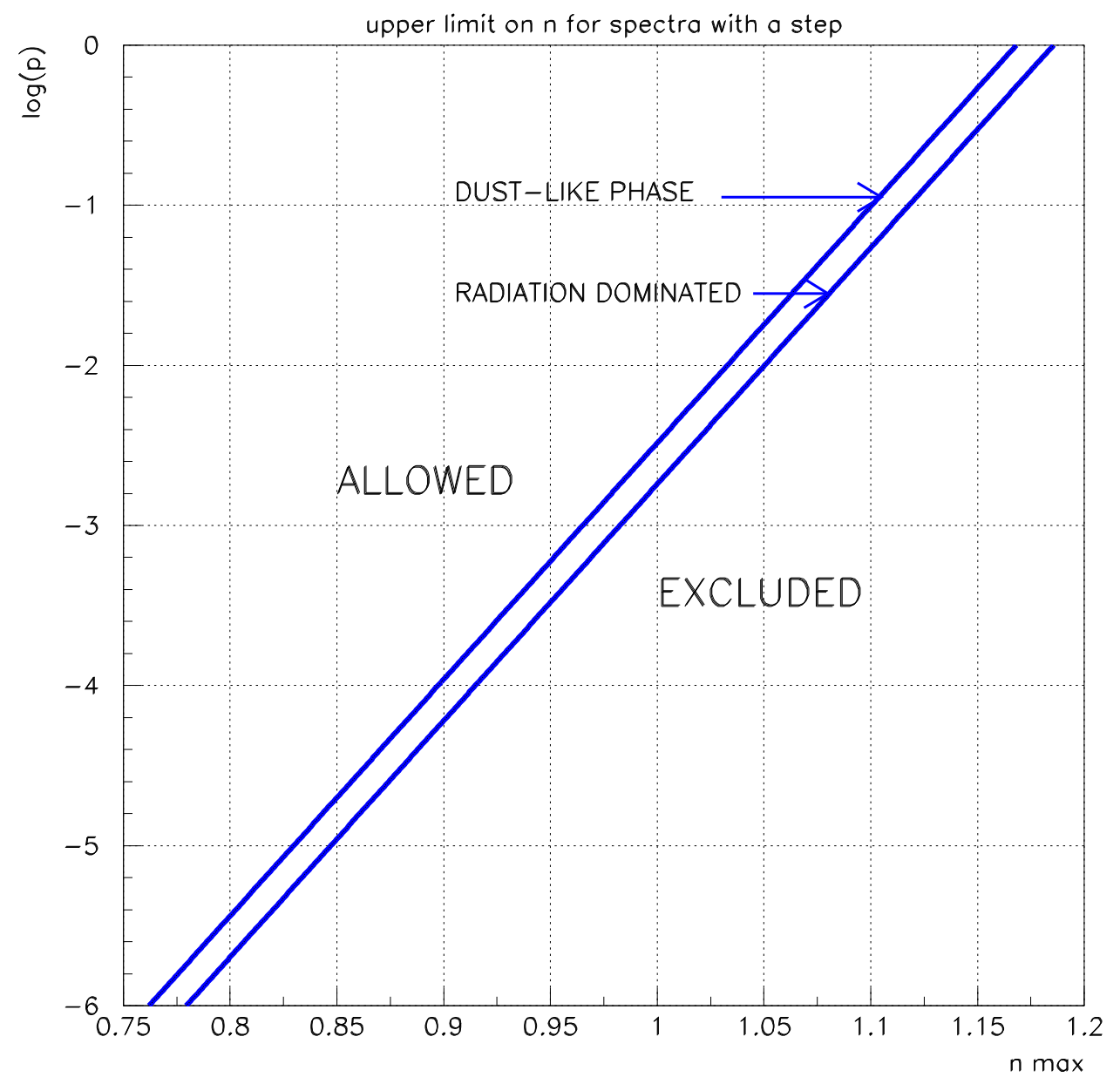

Figure 3: Upper limit on the spectral index of the power spectrum as a function of the amplitude of the step. 
too much power on small scales. In particular, a blue power spectrum or a positive running spectral index are strongly disfavored. Any mechanism which would lead to small scale inhomogeneities with a density contrast above $\sim 0.3$ beyond a tiny fraction of the order of $10^{-12}-10^{-20}$ for masses between $10^{-5}$ and $10^{10} \mathrm{~g}$ are excluded by this analysis. This method offers nontrivial links between the inhomogeneity of the early Universe and the existence of (meta-)stable particles.

It should also be noticed that, as in inflationary cosmology, the equilibrium is established only after reheating at $T<T_{R}$. If $T_{R}$ is as low as [29] $T_{R}<3.8 \times$ $10^{6} \mathrm{GeV}$, superheavy particles with masses $m \gg T_{R}$ and superweakly interacting particles with interaction cross section $\sigma \ll \frac{1}{T_{R} M_{P l}}$ cannot be in equilibrium but can be copiously produced through $\mathrm{PBH}$ evaporation. This property opens a wide range of possible applications for testing particle theory and cosmological scenarios.

\section{Acknowledgment}

M.Kh is grateful to LPSC, CRTBT-CNRS and UJF, Grenoble, France for hospitality.

\section{References}

[1] S. W. Hawking, Mon. Not. R. Astron. Soc. 152 (1971) 75,

B. J. Carr \& S. Hawking, Mon. Not. R. Astron. Soc. 168 (1974) 399

[2] V. Canuto, Mon. Not. R. Astron. Soc. 185 (1978) 721

[3] M. Yu. Khlopov \& A. G. Polnarev, Phys. Lett B 97 (1980) 383,

A. G. Polnarev \& M. Yu. Khlopov, Astr. Zh. 58 (1981) 706

[4] M. Yu. Khlopov, B. A. Malomed \& Ya. B. Zel'dovich, Mon. Not. R. Astron. Soc. 215 (1985) 575

[5] S. W. Hawking, Phys. Lett. B 231 (1989) 237

[6] P. D. Nasel'skii \& Polnarev, Soviet. Astr. 23 (1979) 402

[7] S. W. Hawking, I. G. Moss \& J. M. Stewart, Phys. Rev. D 26 (1982) 2681,

K. Jedamzik \& J.C. Niemeyer, Phys. Rev. D 59 (1999) 124014,

R. V. Konoplich et al., Phys. Atom. Nucl. 62 (1999) 1593,

M. Yu. Khlopov et al., Grav. Cosmol. 6 (2000) 153

[8] D. Blais et al., Phys. Rev. D 67 (2003) 024024

[9] M. Yu. Khlopov, Cosmoparticle Physics, World Scientific (1999) 
[10] B. J. Carr, J. H. Gilbert \& J. E. Lidsey, Phys. Rev. D 50 (1994) 4853,

B. J. Carr \& J. H. MacGibbon, Phys. Rep. 307 (1998) 141,

A. R. Liddle \& A. M. Green, Phys. Rep. 307 (1998) 125

[11] A. G. Polnarev \& M. Yu. Khlopov, Sov. Phys. Uspekhi 28 (1985) 213

[12] S. W. Hawking, Comm. Math. Phys. 43 (1975) 199

[13] V. M. Chechetkin, M. Y. Khlopov \& M. G. Sapozhnikov, Riv. Nuovo Cim. $5 \mathrm{~N} 10(1982) 1$.

[14] M. Y. Khlopov \& V. M. Chechetkin, Fiz. Elem. Chast. Atom. Yadra 18 (1987) 627; English translation: Sov. J. Part. Nucl. 18 (1987) 267.

[15] M. Lemoine, Phys. Lett. B 481 (2000) 333

[16] A. M. Green, Phys. Rev. D 60 (1998) 063516

[17] S. Mujana, K. Sato, Prog. Theor. Phys. 59 (1978) 1012

[18] Ya. B. Zel'dovich et al., Sov. Astron. Lett. 22 (1977) 110

[19] D. Lindley, Mon. Not. R. Astron. Soc. 193 (1980) 593

[20] B. V. Vainer, D. V. Dryzhakova, P. D. Nasselskii, Sov. Astron. Lett. 4 (1978) 185

[21] J. H. MacGibbon, B. Carr, Astrophy. J. 371 (1991) 447

[22] A. Barrau et al., Phys. Lett. B 551 (2003) 218

[23] K. Kohri \& J. Yokoyama, Phys. Rev. D 61 (2000) 023501

[24] A. Barrau et al., astro-ph/0304528, 28th ICRC conference proceedings

[25] A. Barrau et al., Astron. Astrophys. 388 (2002) 676

[26] P. S. Custodio \& J. E. Horvath, Phys. Rev. D 65 (2002) 024023

[27] S. Alexeyev et al., Class. Quantum Grav. 19 (2002) 4431

[28] K. A. Olive, Les Houches Session LXXI, "The Primordial Universe", edited by P. Binetruy et al. (Springer, Berlin, 1999), p. 221

[29] M. Yu. Khlopov et al., Phys. Atom. Nucl. 57 (1994) 1393,

E. V. Sedel'nikov, S. S. Filippov \& M. Yu. Khlopov, Phys. Atomic Nuclei 58 (1995) 235

[30] K. Jedamzik, Phys. Rev. D 70 (2004) 063524,

M. Kawasaki, K. Kohri \& T. Moroi, RESCEU-04/04 astro-ph/0402490 
[31] A. Barrau \& N. Ponthieu, Phys. Rev. D 69 (2004) 105021

[32] C. F. Kolda, Nucl. Phys. Proc. Suppl. 62 (1998) 266

[33] A. G. Polnarev \& M. Yu. Khlopov, Astron. Zh. 59 (1982) 639

[34] K. Jedamzik, M. Lemoine \& G. Moultaka, hep-ph/0506129,

M. Lemoine, G. Moultaka \& K. Jedamzik, hep-ph/0504021

[35] D. N. Spergel et al., Astrophys. J. Suppl. 148 (2003) 175

[36] A. M. Green \& A. R. Liddle, Phys. Rev. D 56 (1997) 6166,

T. Bringmann, C. Kiefer \& D. Polarski, Phys. Rev. D 65 (2002) 024008

[37] H. I. Kim, C. H. Lee. \& J. H. MacGibbon, Phys .Rev. D5 9 (1999) 063004

[38] A. A. Starobinsky, JETP Lett. 55 (1992) 489

[39] J. H. MacGibbon, Nature 329 (1987) 308

[40] A. Barrau et al., Ann. Phys. 13 (2004) 115

[41] A. R. Liddle \& D. H. Lyth, eds., Cosmological inflation and large scale structure (Cambridge University Press, Cambdridge, England, 2000).

[42] M. Kawasaki, M. Yamaguchi \& J. Yokoyama, Phys. Rev. D 68 (2003) 023508

[43] U. Seljak et al., Phys. Rev. D, 71 (2005) 103315

[44] H. V. Peiris et al., Astrophys. J., 148 (2003) 213

[45] J. Khoury, P. J. Steinhardt \& N. Turok, Phys. Rev. Lett 91 (2003) 161301

[46] M. Yu. Khlopov et al. Astron. Lett. 24 (1998) 413,

M. Yu. Khlopov et al., Grav. Cosmol. 2 (1999) S1 